\title{
On application of algebra of quaternions I
}

\author{
Emran Sasi Althabit *, Ismail.M.Mohamed ${ }^{* *}$ \\ * Higher Institute for water Affairs \\ ** Academic of Engineering and Medical Sciences \\ DOI: 10.29322/IJSRP.11.02.2021.p11040 \\ http://dx.doi.org/10.29322/IJSRP.11.02.2021.p11040
}

\begin{abstract}
We introduced in this paper the quaternions concepts and quaternions algebra, Representation of quaternions, transformation of quaternion in space exactly rotation and as application : Quaternions in space.
\end{abstract}

Key words: Quaternion, Complex quaternions , Interpolation of quaternions .

\section{INTRODUCTION}

$\mathrm{T}$ he important of quaternions, appeared in a physics and a Computer programs.

* In physic s, quaternions are correlated to nature of the universe at the level of quantum mechanics. They lead to elegant expression of the Lorentz transformations, which form the basis of the modern theory of relativity.[6]

* In a computer programs transformation of quaternions use for Animation and Simulation, The Quaternion Fourier Transform (QFT) is a powerful tool, its application include face recognition and voice recognition in and robot kinematics as a simulation of nature of the universe.

\section{Hamilton's Quaternions}

Definition1. 1:A quaternion is a four-dimensional complex number that can be used to represent the orientation of a rigid body or coordinate frame in three-dimensional space. The Hamilton's quaternions $\mathbb{H}$ can be generalized to allow coefficients in $\mathbb{R}$ as

$$
\mathbb{H}=\{Q=w+x i+y j+z k: w, x, y, z \in \mathbb{R}\}
$$

Or $\mathbb{H}=\{(w, x, y, z): w, x, y, z \in \mathbb{R}\}$

So that quaternion is the sum of a scalar part $w$ and a vectorial part $(x, y, z) \in \mathbb{R}^{3}$ i.e.,if $\vec{r}=x i+y j+z k$ is position vector in space quaternions is define as

$w+x i+y j+z k \leftrightarrow w+\vec{r}=[w, \vec{r}]$

in simply where $w$ real part $\operatorname{Re}(q)$, and $\vec{r}$ represent imaginary part $=\operatorname{Im}(q)$-vector in

3-dimession space, If $=0$, the quaternion

$q=x i+y j+z k$

is called pure quaternion i.e., A $3 \mathrm{D}$ vector is a pure quaternion whose real part is zero.

where $i, j$ and $k$ are the standard orthonormal basis in $\mathbb{R}^{3}$.

The multiplication for the primitive elements $i, j$ and $k$ is defined by

$i j=-j i=k$

$j k=-k j=i$

$k i=-k i=j$

$i^{2}=j^{2}=k^{2}=i j k=-1$

\begin{tabular}{|c|r|r|r|r|r|r|r|r|}
\hline & $\mathbf{1}$ & $-\mathbf{1}$ & $\mathbf{i}$ & $-\mathbf{i}$ & $\mathbf{j}$ & $-\mathbf{j}$ & $\mathbf{k}$ & $-\mathbf{k}$ \\
\hline $\mathbf{1}$ & 1 & -1 & $\mathrm{i}$ & $-\mathrm{i}$ & $\mathrm{j}$ & $-\mathrm{j}$ & $\mathrm{k}$ & $-\mathrm{k}$ \\
\hline $\mathbf{- 1}$ & -1 & $\mathrm{1}$ & $-\mathrm{i}$ & $\mathrm{i}$ & $-\mathrm{j}$ & $\mathrm{j}$ & $-\mathrm{k}$ & $\mathrm{k}$ \\
\hline $\mathbf{i}$ & $\mathrm{i}$ & $-\mathrm{i}$ & -1 & 1 & $\mathrm{k}$ & $-\mathrm{k}$ & $-\mathrm{j}$ & $\mathrm{j}$ \\
\hline$-\mathbf{i}$ & $-\mathrm{i}$ & $\mathrm{i}$ & 1 & -1 & $-\mathrm{k}$ & $\mathrm{k}$ & $\mathrm{j}$ & $-\mathrm{j}$ \\
\hline $\mathbf{j}$ & $\mathrm{j}$ & $-\mathrm{j}$ & $-\mathrm{k}$ & $\mathrm{k}$ & -1 & 1 & $\mathrm{i}$ & $-\mathrm{i}$ \\
\hline$-\mathbf{j}$ & $-\mathrm{j}$ & $\mathrm{j}$ & $\mathrm{k}$ & $-\mathrm{k}$ & 1 & -1 & $-\mathrm{i}$ & $\mathrm{i}$ \\
\hline $\mathbf{k}$ & $\mathrm{k}$ & $-\mathrm{k}$ & $\mathrm{j}$ & $-\mathrm{j}$ & $-\mathrm{i}$ & $\mathrm{i}$ & -1 & 1 \\
\hline$-\mathbf{k}$ & $-\mathrm{k}$ & $\mathrm{k}$ & $-\mathrm{j}$ & $\mathrm{j}$ & $\mathrm{i}$ & $-\mathrm{i}$ & 1 & -1 \\
\hline
\end{tabular}

Table 1: Multiplication Table for the Quaternion Group. Also called a Cayley Group Table. Conversion order: Row entry first followed by column entry.(Adapted from Weisstein, 1999-2014)

and

$\mathrm{i} \times \mathrm{j}=\mathrm{k}, \mathrm{j} \times \mathrm{k}=\mathrm{i}, \mathrm{k} \times \mathrm{i}=\mathrm{j} \quad(1.5)$

Which are very similar to the cross product of two unit Cartesian vectors.

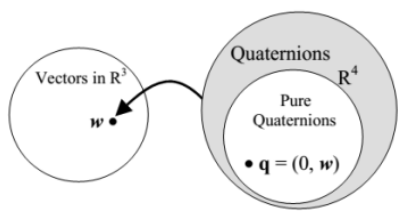

Fig.2 A one-to-one correspondence between pure quaternions and vectors in $\mathbb{R}^{3}[3]$

\section{Octonions}

In the same year in which Hamilton discover the quaternions wrote to his friend John Graves about his discovery Three months later on December $26^{\text {th }}$ Graves wrote to Hamilton about his discovery of a kind of 'double-quaternion' that he called 'octives', today are they are known as octonions. Like quaternions octonions form a division algebra, but unlike quaternions they are not associative. They are related to geometries in 7 and 8 dimensions.[6]

$$
\begin{aligned}
& O=s+e_{1} x_{1}+e_{2} x_{2}+e_{3} x_{3}+e_{4} x_{4}+e_{5} x_{5}+e_{6} x_{6} \\
& +e_{7} x_{7}
\end{aligned}
$$

Mathematically, octonions are the largest normed division algebras. This means that they satisfy $|a b|^{2}=|a|^{2}|b|^{2}$.

When Hamilton discovered quaternions had to give up commutative property in order to preserve the norm; now the associative property has to be given up in order to preserve the norm for the octonions.

A multiplication table for octonions can be made like quaternions as follows: 


\begin{tabular}{|c|c|c|c|c|c|c|c|c|}
\hline & $e_{1}$ & $e_{2}$ & $e_{3}$ & $e_{4}$ & $e_{5}$ & $e_{6}$ & $e_{7}$ & \\
\hline$e_{1}$ & -1 & $e_{4}$ & $e_{7}$ & $-e_{2}$ & $\mathrm{e}_{6}$ & $-e_{5}$ & $-e_{3}$ & \\
\hline$e_{2}$ & $-e_{4}$ & -1 & $e_{5}$ & $e_{1}$ & $-e_{3}$ & $e_{7}$ & $-e_{6}$ & table for Octonions. \\
\hline$e_{3}$ & $-e_{7}$ & $-e_{5}$ & -1 & $e_{6}$ & $e_{7}$ & $-e_{4}$ & $e_{1}$ & (Baez, 2001c) \\
\hline$e_{4}$ & $e_{2}$ & $-e_{1}$ & $-e_{6}$ & -1 & $e_{7}$ & $e_{3}$ & $-e_{5}$ & \\
\hline$e_{5}$ & $-e_{6}$ & $e_{3}$ & $-e_{2}$ & $-e_{7}$ & -1 & $\mathrm{e}_{1}$ & $\mathrm{e}_{4}$ & \\
\hline$e_{6}$ & $e_{5}$ & $-e_{7}$ & $\mathrm{e}_{4}$ & $-e_{3}$ & $-\mathrm{e}_{1}$ & -1 & $e_{2}$ & \\
\hline$e_{7}$ & $e_{3}$ & $e_{6}$ & $-e_{1}$ & $e_{5}$ & $-\mathrm{e}_{4}$ & $-e_{2}$ & -1 & \\
\hline
\end{tabular}

Some of the properties of octonions as they relate to quaternions are the multiplication table for octonions[6]

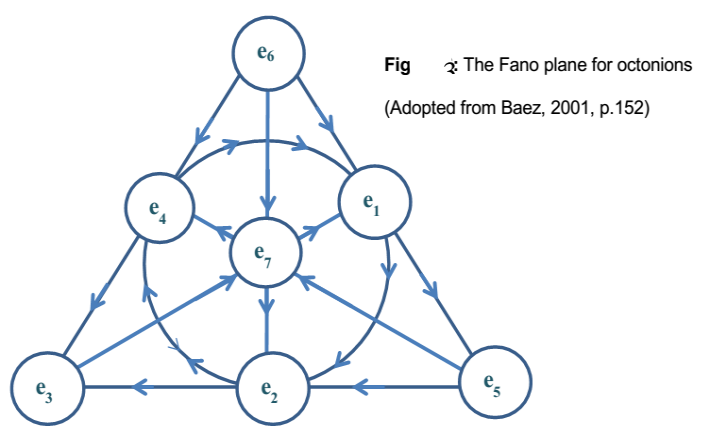

For example: $e_{i}, e_{j}$, and $e_{k}$ can be represented as follows: $e_{i} e_{j}=e_{k} ; e_{j} e_{i}=-e_{k}$. (1.7)

Let 1 be the multiplicative identity.

Let each circle in the diagram be

represented by the relationship $e_{i}^{2}=-1[6]$

2. Quaternions Algebras

The Operations on quaternions 2.1:

Let $Q_{1}$ and $Q_{2}$ be two quaternions, given as

$Q_{1}=w_{1}+x_{1} i+y_{1} j+z_{1} k$

$Q_{2}=w_{2}+x_{2} i+y_{2} j+z_{2} k$, then

1 - The addition and subtraction are define as

$Q_{1} \pm Q_{2}=\left(w_{1}+x_{1} i+y_{1} j+z_{1} k\right) \pm\left(w_{2}+x_{2} i+y_{2} j+z_{2} k\right)$
$\quad=\left(w_{1} \pm w_{2}\right)+\left(x_{1} \pm x_{2}\right) i+\left(y_{1} \pm y_{2}\right) j+\left(z_{1} \pm z_{2}\right) k$

2- The multiplication

From multiplication of the primitive elements $i, j$ and $k$ is defined multiplication of two quaternions $Q_{1}$ and $Q_{1}$ as

$$
\begin{aligned}
Q_{1} Q_{2}= & \left(w_{1}+x_{1} i+y_{1} j+z_{1} k\right)\left(w t_{2}+x_{2} i+y_{2} j+z_{2} k\right) \\
& \left(w_{1} w_{2}-x_{1} x_{2}-y_{1} y_{2}-z_{1} z_{2}\right) \\
& +\left(w t_{1} x_{2}+x_{1} w_{2}+y_{1} z_{2}-z_{1} y_{2}\right) i \\
& +\left(w_{1} y_{2}-x_{1} z_{2}+y_{1} w_{2}+z_{1} x_{2}\right) j \\
+ & \left(w t_{1} z_{2}+x_{1} y_{2}-y_{1} x_{2}-z_{1} w t_{2}\right) k
\end{aligned}
$$

We have $Q_{1} Q_{2} \neq Q_{2} Q_{1}$ i.e., the multiplication of quaternions is not commutative in general . the quaternions is also viewed as $Q=w+\vec{r}$, where

$\vec{r}=x i+y j+z k$, we identify with $3 \mathrm{D}$ vector $(x, y, z)$ so the dot product $(\cdot)$ and cross product $(\times)$ of vectors introduced the familiar notation and so we define the canonical scalar product on III by

$$
\begin{aligned}
& Q_{1} Q_{2}=\left(w_{1}+\vec{r}_{1}\right)\left(w t_{2}+\vec{r}_{2}\right) \\
& =\left(w_{1} w_{2}-\overrightarrow{r_{1}} \cdot \overrightarrow{r_{2}}\right)+w_{1} \overrightarrow{r_{2}}+w_{2} \overrightarrow{r_{1}}+\overrightarrow{r_{1}} \times \overrightarrow{r_{2}}
\end{aligned}
$$

The multiplication of quaternions is not a commutative over a real numbers so that it is called is a non-commutative algebra.
The square of a quaternion is given by

$Q=[w, \vec{r}]$, then

$Q^{2}=[w, \vec{r}][w, \vec{r}]=\left[w^{2}-\vec{r} \cdot \vec{r}, 2 w \vec{r}+\vec{r} \times \vec{r}\right]$

$=\left[w^{2}-\vec{r} \cdot \vec{r}, 2 w \vec{r}\right]$

$Q^{2}=\left[w^{2}-x^{2}-y^{2}-z^{2}, 2 w(x i+y j+z k)\right]$

(2.4)

The square of a pure quaternion is

$Q^{2}=[0, \vec{r}][0, \vec{r}]=[0-\vec{r} \cdot \vec{r}, \vec{r} \times \vec{r}]$

$$
=[-\vec{r}, \vec{r}, 0]=\left[-\left(x^{2}+y^{2}+z^{2}\right), 0\right]
$$

3- The quotient

We represent the quotient $Q_{1} / Q_{2}$ as

$$
Q_{3}=Q_{1} Q_{2}^{-1}=\frac{Q_{1} Q_{2}^{*}}{\left|Q_{2}\right|^{2}}
$$

Algebraic properties 2.2:

(1) Norm:

$|Q|=\sqrt{w^{2}+x^{2}+y^{2}+z^{2}}$

and for two quaternions $\mathrm{p}, \mathrm{q}$ satisfies the norm of product $|\mathbf{Q P}|=|\mathbf{Q}||\boldsymbol{P}|$

(2) Conjugate:

$\begin{aligned} Q^{*} & =(\boldsymbol{w}+\boldsymbol{x} \boldsymbol{i}+\boldsymbol{y} \boldsymbol{j}+\boldsymbol{z} \boldsymbol{k})^{*} \\ & =\boldsymbol{w}-\boldsymbol{x i}-\boldsymbol{y} \boldsymbol{j}-\boldsymbol{z} \boldsymbol{k}\end{aligned}$

and for two quaternions $\mathrm{p}, \mathrm{q}$ satisfies the conjugate of $\operatorname{product}(\boldsymbol{Q P})^{*}=\boldsymbol{P}^{*} \boldsymbol{Q}^{*}$

(3) Multiplicative inverse:

$$
\boldsymbol{Q}^{-\mathbf{1}}=\frac{Q^{*}}{|Q|}
$$

and for two quaternions $\mathrm{P}, \mathrm{Q}$ satisfies the inverse of product

(4) Real part of Q

$$
(P Q)^{-1}=Q^{-1} P^{-1}
$$

(5) The dot product

$$
w=\frac{Q+Q^{*}}{2}
$$

$$
\begin{aligned}
& Q_{1} \cdot Q_{2}=w_{1} w_{2}+x_{1} x_{2}+y_{1} y_{2}+z_{1} z_{2} \\
& =Q_{1} \cdot Q_{2}^{*}
\end{aligned}
$$

(6) The unit quaternion: has norm 1, i.e., $|Q|=w^{2}+x^{2}+y^{2}+$ $\boldsymbol{y}^{\mathbf{2}}=\mathbf{1} \quad$ (2.11)

and inverse of unit quaternion $Q^{-1}=Q^{*}$

and it can be represented by

$Q=\cos \theta+\widehat{u} \sin \theta$

Where $\widehat{\mathbf{u}}$ is the vector has length 1 . However, observe that the quaternion product $\widehat{\mathbf{u}} \widehat{\mathbf{u}}=\mathbf{- 1}$

Note in general : a pure unit quaternion is a square root of $\mathbf{- 1}$, such as imaginary units and Euler's identity of quaternion The unit Quaternion is normalised with respect to the Hermitian inner product, where $I_{2}$ is the $2 \times 2$ identity matrix.

A quaternion with a unit norm is called a normalised quaternion

$$
Q^{\prime}=\frac{Q}{|Q|}=\frac{Q}{\sqrt{w^{2}+|\overrightarrow{\boldsymbol{r}}|^{2}}}
$$

(7) The unit length Euler's identity for quaternions $\exp (\widehat{\mathbf{u}} \theta)=\cos \theta+\widehat{\mathbf{u}} \sin \theta$

where the exponential on the left-hand side is evaluated by

$Q^{n}=(\cos \theta+\widehat{\mathbf{u}} \sin \theta)^{n}$

$$
=\cos (n \theta)+\widehat{u} \sin (n \theta)
$$$$
=\exp (\widehat{\mathbf{u}} \boldsymbol{n} \theta)
$$

(8) It is also possible to define the logarithm of a unit quaternion, $\ln Q=\ln (\cos \theta+\widehat{u} \sin \theta)$ 


$$
=\ln \exp (\widehat{\mathbf{u}} \theta)=\widehat{\mathbf{u}} \boldsymbol{\theta}
$$

The quaternions can be thought of as a choice of a group structure (9) The quaternion polar coordinate representation is

$$
\begin{aligned}
Q & =|Q|(\cos \theta+\widehat{u} \sin \theta) \\
& =|Q| \exp (\hat{u} \theta)
\end{aligned}
$$

(10) The multiplication of two vectors

If we multiply together two imaginary quaternions $\boldsymbol{P}, Q \in \mathbb{H}_{\mathbf{p}}$, where $\mathbb{H}_{\mathbf{p}}$ is set of pure quaternions we obtain a quaternionic version of the scalar product and vector product on $\mathbb{R}^{3}$ as follows[10]

(2.18)

$$
\boldsymbol{P} Q=-\boldsymbol{P} \cdot Q+\boldsymbol{P} \wedge Q \in \mathbb{R} \oplus \mathbb{H}_{\mathbf{p}} \cong \mathbb{H}
$$

Others algebraic properties

The set of quaternions satisfy some other algerbic properties that worth mentioning

* The set of quaternions is an abelian group $(\mathbb{H},+)$ under quaternion addition .

* The set of quaternions is not abelian rig $(\mathbb{H},+, \cdot)$ where + and $\cdot$ are quaternion addition and multiplication .[14]

\section{Matrix representation of quaternion}

The are at least two ways for representation of quaternions as matrices in such way that quaternions addition and multiplication correspond to matrix addition and multiplication .One is use $2 \times 2$ complex matrices , and the other is to use $4 \times 4$ real matrices. In each case, the representation given is one of a family of lineally related representations .[13]

The $4 \times 4$ real matrix representation 3.1

The quaternion is written in $4 \times 4$ real matrix notation as follows

$$
[w, x \boldsymbol{i}+y \boldsymbol{j}+z \boldsymbol{k}] \mapsto \boldsymbol{L}_{Q}=\left[\begin{array}{cccc}
\boldsymbol{w} & -\boldsymbol{x} & -\boldsymbol{y} & -\boldsymbol{z} \\
\boldsymbol{x} & \boldsymbol{w} & \boldsymbol{z} & -\boldsymbol{y} \\
\boldsymbol{y} & -\boldsymbol{z} & \boldsymbol{w} & \boldsymbol{x} \\
\boldsymbol{z} & \boldsymbol{y} & -\boldsymbol{x} & \boldsymbol{w}
\end{array}\right]
$$

Orthogonal Matrix

We can demonstrate that the unit-norm quaternion matrix is orthogonal the product of $\mathrm{L}_{\mathrm{Q}}$ with its transpose $\mathrm{L}_{Q^{*}}=\mathrm{L}_{\mathrm{Q}}^{\mathrm{T}}$ equals the identity matrix $I$ (where $\mathrm{I}$ is $4 \times 4$ marix).

The $2 \times 2$ complex matrix representation 3.2

We switch from thinking of $\mathbb{H}$ as 4-D with real scalars and basis $, \boldsymbol{i}, \boldsymbol{j}, \boldsymbol{k}$, to being 2 -D with complex scalars and basis $\mathbf{1}, \boldsymbol{j}$ by writing $Q=w+x \boldsymbol{i}+\boldsymbol{y} \boldsymbol{j}+\boldsymbol{z} \boldsymbol{k}$

$$
\begin{aligned}
& =(w+x i)+(y+z i) j \\
& =\alpha+\beta j
\end{aligned}
$$

Where the map $j: \alpha+\beta j \mapsto-\bar{\beta}+\bar{\alpha} j$

is a conjugate-linear involution of $\mathbb{C}^{2}$ with $j^{2}=-1$ This identification $\mathbb{H} \cong \mathbb{C}^{2}$. [10]

Having written this down, it is easy to form the map: $\mathbb{H} \rightarrow \mathcal{H} \subset$ $\operatorname{Mat}(2, \mathbb{C})$

$$
\begin{array}{r}
Q=\alpha+\boldsymbol{\beta} \mathbf{j} \mapsto M_{Q}=\left[\begin{array}{cc}
\alpha & \beta \\
-\bar{\beta} & \bar{\alpha}
\end{array}\right] \\
\mathbf{1}=\left[\begin{array}{ll}
\mathbf{1} & \mathbf{0} \\
\mathbf{0} & \mathbf{1}
\end{array}\right], \boldsymbol{i}=\left[\begin{array}{cc}
\boldsymbol{i} & \mathbf{0} \\
\mathbf{0} & -\boldsymbol{i}
\end{array}\right] \\
j=\left[\begin{array}{cr}
\mathbf{0} & \mathbf{1} \\
-\mathbf{1} & \mathbf{0}
\end{array}\right], k=\left[\begin{array}{ll}
\mathbf{0} & \boldsymbol{i} \\
\boldsymbol{i} & \mathbf{0}
\end{array}\right]
\end{array}
$$

The quaternion algebra can thus be realised as a real subalgebra of $\operatorname{Mat}(2, \mathbb{C})$, using the icidentifations

$$
i=\left[\begin{array}{cc}
i & 0 \\
0 & -i
\end{array}\right]=\left[\begin{array}{cc}
1 & 0 \\
0 & -1
\end{array}\right] i
$$

$$
\begin{gathered}
j=\left[\begin{array}{cc}
0 & 1 \\
-1 & 0
\end{array}\right]=\left[\begin{array}{cc}
0 & i \\
-i & 0
\end{array}\right] i \\
k=\left[\begin{array}{ll}
0 & i \\
i & 0
\end{array}\right]=\left[\begin{array}{cc}
0 & 1 \\
1 & 0
\end{array}\right] i
\end{gathered}
$$

so that if we define the matrices $\sigma_{1}, \sigma_{2}, \sigma_{3}$ such that

$\sigma_{1}=\left[\begin{array}{cc}1 & 0 \\ 0 & -1\end{array}\right], \sigma_{2}=\left[\begin{array}{cc}0 & -i \\ i & 0\end{array}\right], \sigma_{3}=\left[\begin{array}{ll}0 & 1 \\ 1 & 0\end{array}\right]$ (3.3)

we can write

$Q=\boldsymbol{w} \mathbf{1}+\boldsymbol{x i}+\boldsymbol{y} \boldsymbol{j}+\boldsymbol{z k}$

$=w \mathbf{1}+\boldsymbol{i}\left(\mathbf{z} \sigma_{1}+\boldsymbol{y} \boldsymbol{\sigma}_{2}+\boldsymbol{x} \boldsymbol{\sigma}_{3}\right)$

The matrices $\sigma_{1}, \sigma_{2}, \sigma_{3}$ are called the Pauli spin matrices.

Note that the irtraces are null and that they are Hermitian (recall that a complex matrix is Hermitian if it is equal to the transpose of its conjugate, i.e., $\boldsymbol{A}^{*}=\boldsymbol{A}$ ). If we let $\boldsymbol{e}_{\mathbf{0}}=\boldsymbol{t}$,

$\boldsymbol{e}_{\mathbf{1}}=\boldsymbol{z}, \boldsymbol{e}_{\mathbf{2}}=\boldsymbol{y}$ and $\boldsymbol{e}_{\mathbf{3}}=\boldsymbol{x}$, then $\boldsymbol{q}$ can be written as

$Q=e_{0} \mathbf{1}+i\left(e_{1} \sigma_{1}+e_{2} \sigma_{2}+e_{3} \sigma_{3}\right)(3.5)$

And $\boldsymbol{e}_{\mathbf{0}}, \boldsymbol{e}_{\mathbf{1}}, \boldsymbol{e}_{\mathbf{2}}, \boldsymbol{e}_{\mathbf{3}}$ are called theEuler parametersof the rotation specified by $q$

$|Q|=1$, then we can write also

$Q=\cos 1 / 2 \theta+i \sin 1 / 2 \theta\left(\beta \sigma_{3}+\gamma \sigma_{2}+\delta \sigma_{1}\right)$

(3.6)

Where

$$
(\boldsymbol{\beta}, \boldsymbol{\gamma}, \boldsymbol{\delta})=\frac{1}{\sin 1 / 2 \theta}(x, y, z)
$$

Letting $\boldsymbol{A}=\boldsymbol{\beta} \boldsymbol{\sigma}_{\mathbf{3}}+\boldsymbol{\gamma} \boldsymbol{\sigma}_{\mathbf{2}}+\boldsymbol{\delta} \boldsymbol{\sigma}_{\mathbf{1}}$, it can be shown that

$\exp (\boldsymbol{i \theta A})=\cos \theta 1+\boldsymbol{i} \sin \theta \boldsymbol{A}$ (3.7)

Note that : The exponential is the usual exponential of matrices, i.e., for a square

$\boldsymbol{n} \times \boldsymbol{n}$ matrix $M$

$$
\exp (A \theta)=I_{n}+\sum_{k \geq 1} \frac{(A \theta)^{k}}{k !}
$$

Note that the rows (and columns) of such matrices are vectors in $\mathbb{C}^{2}$ that are orthogonal with respect to the Hermitian inner product of $\mathbb{C}^{2}$ given by

$\mathcal{H} \subset$ Furthermore, their norm is

$$
\sqrt{\alpha \bar{\alpha}++\beta \bar{\beta}}=\sqrt{w^{2}+x^{2}+y^{2}+z^{2}}
$$

and the determinant of $\boldsymbol{A}$ is

$$
w^{2}+x^{2}+y^{2}+z^{2}
$$

The vector space $\mathbb{R}^{3}$ is a Lie algebra 3.3

If we define the Lie bracket on $\mathbb{R}^{3}$ as the usual cross product $\boldsymbol{u} \times$ $\mathbf{v}$ of vectors. Then the Lie algebra of $\boldsymbol{S U}(2)$ is isomorphic to $\left(\mathbb{R}^{3}, \times\right)$

and the exponential map can be viewed as a map exp: $\left(\mathbb{R}^{3}, x\right) \rightarrow$ $\boldsymbol{S U}(2)$ given bythe formula

$$
\boldsymbol{e x p}(\boldsymbol{\theta} \widehat{\mathbf{u}})=[\cos 1 / 2 \theta, \sin 1 / 2 \theta \widehat{\mathbf{u}}](3.8)
$$

a vector is rotated about the axis $\widehat{\mathbf{u}}$ by an angle $\boldsymbol{\theta}$. This half-angle representation was

discovered by Rodrigues .

Note that the squared norm $Q Q^{*}$ of a quaternion $\mathrm{q}$ is the same as the determinant of the matrix $\mathbf{M}_{Q} \in \mathbb{H}$. The isomorphism $\mathbf{M}_{\mathbf{Q}}$ gives an easy way to deduce that $\mathbb{H}$ is an associative division algebra; the inverse of any nonzero matrix $\mathbf{A} \in \mathbb{H}$ is also in $\mathbb{H}$, and the only matrix in $\mathbb{H}$ whose determinant is zero is the zero matrix i.e., the quaternion algebra can be viewed as a union of complex planes, so the hyperbolic quaternion algebra is a union of split-complex number planes sharing the same real line . 
4. complex quaternion

Definition.4.1: A complex quaternion is an element of the form

where

$$
Q=A_{0}+A_{1} e_{1}+A_{2} e_{2}+A_{3} e_{3}
$$

$\boldsymbol{A}_{\mathbf{0}}, \boldsymbol{A}_{1}, \boldsymbol{A}_{2}$ and $\boldsymbol{A}_{3}$ complex numbers and $\boldsymbol{e}_{1}, \boldsymbol{e}_{2} \boldsymbol{e}_{3}$ are quaternionic units which satisfy the equalities

$e_{1}^{2}=e_{2}^{2}=e_{3}^{2}=e_{1} e_{2} e_{3}=-1$,

$e_{1} e_{2}=-e_{2} e_{1}=e_{3}$

$e_{2} e_{3}=-e_{3} e_{2}=e_{1}$,

$e_{3} e_{1}=-e_{1} e_{3}=e_{2}$

As a consequence of this dentition, a complex quaternion $Q$ can also be written

$\boldsymbol{Q}+\boldsymbol{i} \boldsymbol{Q}^{*}$

Where $\boldsymbol{Q}, \boldsymbol{Q}^{*}$ are real quaternions and $\boldsymbol{i}^{2}=-\mathbf{1}$.

The set of all complex quaternions is denoted by $\mathbb{H}_{\mathbb{C}}$. Also, a complex quaternion $\mathbf{Q}$ is a sum of a scalar and a vector, called scalar part $S_{Q}=A_{0}$ and vector part

$\mathrm{V}_{\mathrm{Q}}=\boldsymbol{A}_{1} \boldsymbol{e}_{1}+\boldsymbol{A}_{2} \boldsymbol{e}_{2}+\boldsymbol{A}_{3} \boldsymbol{e}_{3}$

If $\boldsymbol{S}_{Q}=\mathbf{0}$; then $\mathrm{Q}$ is called pure complex quaternion, we may call it a complex vector. The algebra of biquaternions can be considered as a tensor product $\mathbb{C} \otimes \mathbb{H}$

(taken over the reals)where $\mathbb{C}$ is the field of complex numbers and $\mathbb{H}$ is the algebra of real quaternions. In other words, the biquaternions are just the complexication of the real quaternions. The product of two complex quaternions $\boldsymbol{Q}=\boldsymbol{S}_{\boldsymbol{Q}}+\overrightarrow{\mathbf{V}}_{\mathbf{Q}}$ and $\boldsymbol{P}=$ $S_{P}+\overrightarrow{\mathbf{V}}_{\mathbf{P}}$ and If a complex quaternion is looked at as a fourdimensional vector, the complex

quaternion product can be describe by a matrix-vector products as:

$$
Q P=\left[\begin{array}{cccc}
A_{0} & -A_{1} & -A_{2} & -A_{3} \\
A_{1} & A_{0} & -A_{3} & A_{2} \\
A_{2} & A_{3} & A_{0} & -A_{1} \\
A_{3} & -A_{2} & A_{1} & A_{1}
\end{array}\right]\left[\begin{array}{l}
B_{0} \\
B_{1} \\
B_{2} \\
B_{3}
\end{array}\right]
$$

From now one, we will identify the quaternion with the "complex" quaternion .[4]

\section{Quaternions in Space}

In 1898 the German mathematician, Adolf Hurwitz (18591919), proved that the product of the sum of $\boldsymbol{n}$ squares by the sum of $\boldsymbol{n}$ squares is the sum of $\mathrm{n}$ squares only when $\boldsymbol{n}$ is equal to 1, 2, 4 and 8 , which are represented by the reals, complex numbers, quaternions and octonions. This is known as 'Hurwitz's Theorem' or the ' $1,2,4,8$ Theorem'. No other system is possible, which shows how important quaternions are within the realm of mathematics. Consequently, Hamilton's

search for a system of triples was futile, because there is no threesquare identity.

Aunit-norm quaternion $\boldsymbol{Q}$ used to rotate

a vector $\overrightarrow{\mathbf{v}}$ stored as a pure quaternion $\mathrm{p}$ as the product $\boldsymbol{Q P}$, where $\boldsymbol{Q}=[\boldsymbol{w}, \lambda \hat{\mathbf{v}}], \boldsymbol{w}, \lambda \in \mathbb{R}, \hat{\mathbf{v}} \in \mathbb{R}^{3}$,

$|\hat{\mathbf{v}}|=\mathbf{1}, w^{2}+\lambda^{2}=\mathbf{1}$.

and the pure quaternion $\boldsymbol{P}$ stores the vector $\overrightarrow{\boldsymbol{r}}$ to be rotated:

$\boldsymbol{P}=[\mathbf{0}, \overrightarrow{\boldsymbol{r}}], \boldsymbol{p} \in \mathbb{R}^{\mathbf{3}}$

We compute the product $\boldsymbol{P}^{\prime}=\boldsymbol{Q P}$

and examine the vector part of $\mathrm{p}$

to see if $\boldsymbol{P}$ is rotated:

$\boldsymbol{P}^{\prime}=\boldsymbol{Q P}=[\boldsymbol{w}, \lambda \hat{\mathbf{v}}][\mathbf{0}, \overrightarrow{\boldsymbol{r}}]$

$=[-\lambda \hat{v} \cdot \vec{r}, w \vec{r}+\lambda \hat{v} \times \vec{r}]$
As we see from (4.2) that the result is a general quaternion with a scalar and a vector component.

As special case: If $\hat{\mathbf{v}}$ perpendicular to $\overrightarrow{\boldsymbol{r}}$, the dot product term $\lambda \hat{\boldsymbol{v}} . \overrightarrow{\boldsymbol{r}}$ in (4.2) vanish, and we have left with the pure quaternion

$$
\boldsymbol{P}^{\prime}=[\mathbf{0}, \boldsymbol{w} \overrightarrow{\boldsymbol{r}}+\lambda \hat{\mathbf{v}} \times \overrightarrow{\boldsymbol{r}}] \quad(4.3)
$$

$\overrightarrow{\boldsymbol{r}}$ is perpendicular to $\hat{\mathbf{v}}$, and $\hat{\mathbf{v}} \times \overrightarrow{\boldsymbol{r}}$ is perpendicular to the plane containing $\overrightarrow{\boldsymbol{r}}$ and $\hat{\mathbf{v}}$. Now because $\hat{\mathbf{v}}$ is a unit vector.

In general Case: We begin by defining a unit-norm quaternion $\boldsymbol{Q}$ : $\boldsymbol{Q}=[\boldsymbol{w}, \lambda \hat{\mathbf{v}}]$ where $\boldsymbol{w}^{2}+\lambda^{2}=\mathbf{1}$, The vector $\overrightarrow{\boldsymbol{r}}$ to be rotated is encoded as a pure quaternion $\boldsymbol{P}=[\mathbf{0}, \overrightarrow{\boldsymbol{r}}]$, and the inverse quaternion $Q^{-1}=[w,-\lambda \hat{v}]$.

Therefore, the product $\boldsymbol{Q P} \boldsymbol{Q}^{\mathbf{- 1}}$ is

$$
\begin{aligned}
Q P Q^{-1} & =[w, \lambda \hat{v}][0, \vec{r}][t,-\lambda \hat{v}] \\
& =[-\lambda \hat{v} . \vec{r}, w \vec{r}+\lambda \hat{v} \times \vec{r}][w,-\lambda \hat{v}] \\
= & {\left[0,2 \lambda^{2}(\hat{v} . \vec{r}) \hat{v}+\left(t^{2}-\lambda^{2}\right) \vec{r}+2 \lambda t \hat{v} \times \vec{r}\right](4.4) }
\end{aligned}
$$

Now, if we want this product to actually rotate the vector by $\theta$, then we must build this in from the outset by halving $\theta$ in $\boldsymbol{Q}$.

Hamilton calls the angle $(0 \leq \theta \leq \pi)$ the angle of , and calls the unit vector $\hat{v}$ the axis of $\boldsymbol{Q}$.

Theorem: For any unit quaternion

$$
\boldsymbol{Q}=[w, \overrightarrow{\mathrm{r}}]=\cos 1 / 2 \theta+\hat{\mathrm{v}} \sin 1 / 2 \theta
$$

and for any vector $\overrightarrow{\mathrm{v}} \in \mathbb{R}^{3}$ the action of operator $L_{q}$ on $\overrightarrow{\mathrm{v}}$ is equivalent to rotation of the vector through angle $\theta$ about $\hat{v}$ as the axis of rotation

$$
L_{Q}(\vec{v})=Q \vec{v} Q^{*}
$$

We substitute the unit quaternion in this form to obtain the resulting vector form rotating a vector $\vec{v}$ about the axis $\hat{v}$ through $\theta$ :

$L_{Q}(\overrightarrow{\mathrm{v}})=\boldsymbol{Q P} \boldsymbol{Q}^{-1}=$

$[0,(1-\cos \theta)(\hat{v} \cdot \vec{r}) \hat{\mathbf{v}}+\cos \theta \vec{r}+\sin \theta \hat{\mathbf{v}} \times \overrightarrow{\boldsymbol{r}}]$ (4.4)

The product $\boldsymbol{Q P} \boldsymbol{Q}^{-\mathbf{1}}$ was discovered by Hamilton who failed to publish the result. Cayley, also discovered the product and published the result in 1845 [8]. However,

Altmann notes that "in Cayley's collected papers he concedes priority to Hamilton" [2], which was a nice gesture. However, the person who had recognised the importance of the half-angle parameters in (3.8) before Hamilton and Cayley was Rodrigueswho published a solution that was not seen by Hamilton, but apparently, was seen by Cayley.

Quaternions in Matrix Form of $\boldsymbol{Q P} \boldsymbol{Q}^{\mathbf{- 1}}$.

The are two methods: the first is a simple vectorial method which translates the vector equation representing $\boldsymbol{Q} \boldsymbol{P} \boldsymbol{Q}^{\mathbf{- 1}}$ directly into matrix form. The second method uses matrix algebra to develop a rather cunning solution.

Vector Method

For the vector method it is convenient to describe the unit-norm quaternion as

$\boldsymbol{Q}=[\boldsymbol{w}, \overrightarrow{\mathbf{v}}]=[\boldsymbol{w}, x \boldsymbol{i}+y \boldsymbol{j}+z \boldsymbol{k}]$, where $\boldsymbol{t}^{2}+|\overrightarrow{\mathbf{v}}|^{2}=\mathbf{1}$

and the pure quaternion as

$$
P=[\mathbf{0}, \vec{u}]=\left[0, \mathbf{u}_{x} \boldsymbol{i}+\mathbf{u}_{y} \boldsymbol{j}+\mathbf{u}_{z} k\right]
$$

A simple way to compute $\boldsymbol{Q P} \boldsymbol{Q}^{-\mathbf{1}}$ is to use (4.3) and substitute $|\overrightarrow{\mathbf{v}}|$ for $\lambda$

$$
\begin{aligned}
& Q P Q^{-1}=\left[0,2 \lambda^{2}(\hat{v} \cdot \vec{u}) \hat{v}+\left(w^{2}-\lambda^{2}\right) \vec{u}+2 \lambda w \hat{v} \times \vec{u}\right] \\
& \quad=\left[0,2|\vec{v}|^{2}(\hat{v} . \vec{u}) \hat{v}+\left(w^{2}-|\vec{v}|^{2}\right) \vec{u}+2|\vec{v}| w \hat{v} \times \vec{u}\right]
\end{aligned}
$$

Next, we substitute $\overrightarrow{\mathbf{v}}$ for $|\overrightarrow{\mathbf{v}}| \hat{\mathbf{v}}$ :

$$
Q P Q^{-1}=\left[0,2(\vec{v} . \vec{u}) \vec{v}+\left(w^{2}-|\vec{v}|^{2}\right) \vec{u}+2 w \vec{v} \times \vec{u}\right]
$$


Since we are working with unit-norm quaternions to prevent scaling

Therefore,

$$
w^{2}+|\overrightarrow{\mathbf{v}}|^{2}=1 \Rightarrow w^{2}-|\overrightarrow{\mathbf{v}}|^{2}=2 w^{2}-1
$$

$$
Q P Q^{-1}=\left[0,2(\vec{v} \cdot \vec{u}) \vec{v}+\left(2 w^{2}-1\right) \vec{u}+2 w \vec{v} \times \vec{u}\right]
$$

If we let $\boldsymbol{P}^{\prime}=\boldsymbol{Q P} \boldsymbol{P} \boldsymbol{Q}^{\mathbf{- 1}}$, which is a pure quaternion, we have

$[0, \vec{u}]=\left[0,2(\vec{v} . \vec{u}) \vec{v}+\left(2 w^{2}-1\right) \vec{u}+2 w \vec{v} \times \vec{u}\right]$

$\vec{u}=\mathbf{2}(\overrightarrow{\mathbf{v}} \cdot \overrightarrow{\boldsymbol{u}}) \overrightarrow{\mathbf{v}}+\left(\mathbf{2} \boldsymbol{w}^{2}-\mathbf{1}\right) \overrightarrow{\boldsymbol{u}}+\mathbf{2} \boldsymbol{w} \overrightarrow{\mathbf{v}} \times \overrightarrow{\boldsymbol{u}}$

We are only interested in the rotated vector $\vec{v}$ comprising the three terms $\mathbf{2}(\overrightarrow{\mathbf{v}} . \overrightarrow{\boldsymbol{u}}) \overrightarrow{\mathbf{v}}$,

$\left(\mathbf{2} \boldsymbol{w}^{2}-\mathbf{1}\right) \overrightarrow{\boldsymbol{u}}, \mathbf{2} \boldsymbol{w} \overrightarrow{\boldsymbol{v}} \times \overrightarrow{\boldsymbol{u}}$, which can be represented by three individual matrices and

summed together.

$$
\begin{aligned}
& \mathbf{2}(\overrightarrow{\mathbf{v}} \cdot \overrightarrow{\boldsymbol{u}}) \overrightarrow{\mathbf{v}}=\mathbf{2}\left(x \mathbf{u}_{x}+y \mathbf{u}_{y}+\mathbf{z u}_{z}\right)(x \boldsymbol{i}+y \boldsymbol{j}+z \boldsymbol{k}) \\
& {\left[\begin{array}{ccc}
\mathbf{2} x^{2} & x y & x z \\
x y & \mathbf{2} y^{2} & y z \\
x z & y z & \mathbf{2} z^{2}
\end{array}\right]\left[\begin{array}{l}
\boldsymbol{u}_{\boldsymbol{x}} \\
\boldsymbol{u}_{y} \\
\boldsymbol{u}_{z}
\end{array}\right]} \\
& \left(2 w^{2}-1\right) \vec{u}=\left(2 w^{2}-1\right) u_{x} i+\left(2 w^{2}-1\right) u_{y} j \\
& +\left(2 w^{2}-1\right) u_{z} k \\
& \left(2 w^{2}-1\right) \vec{u}=\left[\begin{array}{ccc}
2 w^{2}-1 & 0 & 0 \\
0 & 2 w^{2}-1 & 0 \\
0 & 0 & 2 w^{2}-1
\end{array}\right]\left[\begin{array}{l}
u_{x} \\
u_{y} \\
u_{z}
\end{array}\right]
\end{aligned}
$$

$2 w \overrightarrow{\mathbf{v}} \times \overrightarrow{\boldsymbol{u}}=2 w\left(\left(\mathbf{u}_{z} \boldsymbol{y}-\mathbf{z u _ { y }}\right) \boldsymbol{i}+\left(\mathbf{u}_{x} z-z \mathbf{u}_{x}\right) \boldsymbol{j}\right.$

$$
\begin{gathered}
\left.+\left(u_{y} x-x u_{y}\right) k\right) \\
\left(2 w^{2}-1\right) \vec{u}=\left[\begin{array}{ccc}
0 & -2 w z & 2 w y \\
2 w z & 0 & -2 w x \\
-2 w y & 2 w x & 0
\end{array}\right]\left[\begin{array}{l}
u_{x} \\
u_{y} \\
u_{z}
\end{array}\right]
\end{gathered}
$$

Adding these matrices together: $\vec{u}=Q \vec{v} Q^{*}$

$$
\begin{aligned}
& {\left[\begin{array}{ccc}
\mathbf{2}\left(\boldsymbol{w}^{2}+x^{2}\right)-\mathbf{1} & \mathbf{2}(x y-\boldsymbol{w} z) & \mathbf{2}(x z+\boldsymbol{w} y) \\
\mathbf{2}(x y+\boldsymbol{w} z) & \mathbf{2}\left(\boldsymbol{w}^{2}+y^{2}\right)-\mathbf{1} & \mathbf{2}(y z-\boldsymbol{w} x) \\
\mathbf{2}(x z-\boldsymbol{w} y) & \mathbf{2}(y z+\boldsymbol{w} x) & \mathbf{2}\left(\boldsymbol{w}^{2}+z^{2}\right)-\mathbf{1}
\end{array}\right]\left[\begin{array}{l}
\boldsymbol{u}_{x} \\
\boldsymbol{u}_{y} \\
\boldsymbol{u}_{z}
\end{array}\right]} \\
& \text { Or } \vec{u}=Q \vec{v} Q^{*} \\
& {\left[\begin{array}{ccc}
\mathbf{1}-\mathbf{2}\left(y^{2}+z^{2}\right) & \mathbf{2}(x y-\boldsymbol{w} z) & \mathbf{2}(x z+\boldsymbol{w} y) \\
\mathbf{2}(x y+\boldsymbol{w} z) & \mathbf{1}-\mathbf{2}\left(x^{2}+z^{2}\right) & \mathbf{2}(y z-\boldsymbol{w} x) \\
\mathbf{2}(x z-\boldsymbol{w} y) & \mathbf{2}(y z+\boldsymbol{w} x) & \mathbf{1}-\mathbf{2}\left(x^{2}+y^{2}\right)
\end{array}\right]\left[\begin{array}{l}
\boldsymbol{u}_{\boldsymbol{x}} \\
\boldsymbol{u}_{\boldsymbol{y}} \\
\boldsymbol{u}_{z}
\end{array}\right] \text { (4.7) }} \\
& \text { Or } \vec{u}=Q \vec{v} Q^{*} \\
& {\left[\begin{array}{ccc}
w^{2}+x^{2}-y^{2}-z^{2} & \mathbf{2}(x y-\boldsymbol{w z}) & \mathbf{2}(x z+\boldsymbol{w} \boldsymbol{y}) \\
\mathbf{2}(x y+\boldsymbol{w z}) & w^{2}-x^{2}+y^{2}-z^{2} & \mathbf{2}(y z-\boldsymbol{w x}) \\
\mathbf{2}(x z-\boldsymbol{w y}) & \mathbf{2}(y z+\boldsymbol{w} \boldsymbol{x}) & w^{2}-x^{2}-y^{2}+z^{2}
\end{array}\right][}
\end{aligned}
$$

(4.8)

If we reverse the product. To compute the vector part of $\boldsymbol{Q}^{-\mathbf{1}} \mathbf{P Q}$ all that we have to do is reverse the sign of $\overrightarrow{\mathbf{v}} \times \overrightarrow{\boldsymbol{u}}, \vec{u}=Q^{*} \vec{v} Q$

$$
\begin{aligned}
& {\left[\begin{array}{ccc}
\mathbf{2}\left(\boldsymbol{w}^{\mathbf{2}}+x^{2}\right) \mathbf{- 1} & \mathbf{2}(x y+\boldsymbol{w} z) & \mathbf{2}(x z-\boldsymbol{w} y) \\
\mathbf{2}(x y-\boldsymbol{w} z) & \mathbf{2}\left(\boldsymbol{w}^{\mathbf{2}}+y^{2}\right)-\mathbf{1} & \mathbf{2}(y z+\boldsymbol{w} x) \\
\mathbf{2}(x z+\boldsymbol{w} y) & \mathbf{2}(y z-\boldsymbol{w} x) & \mathbf{2}\left(\boldsymbol{w}^{2}+z^{2}\right)-\mathbf{1}
\end{array}\right]\left[\begin{array}{l}
\boldsymbol{u}_{x} \\
\boldsymbol{u}_{y} \\
\boldsymbol{u}_{z}
\end{array}\right]} \\
& \text { Or } \vec{u}=Q^{*} \vec{v} Q \\
& {\left[\begin{array}{ccc}
\mathbf{1}-\mathbf{2}\left(y^{2}+z^{2}\right) & \mathbf{2}(x y+\boldsymbol{w} z) & \mathbf{2}(x z-\boldsymbol{w} y) \\
\mathbf{2}(x y-\boldsymbol{w} z) & \mathbf{1}-\mathbf{2}\left(x^{2}+z^{2}\right) & \mathbf{2}(y z+\boldsymbol{w} x) \\
\mathbf{2}(x z+\boldsymbol{w} y) & \mathbf{2}(y z-\boldsymbol{w} x) & \mathbf{1}-\mathbf{2}\left(x^{2}+y^{2}\right)
\end{array}\right]\left[\begin{array}{l}
\boldsymbol{u}_{x} \\
\boldsymbol{u}_{y} \\
\boldsymbol{u}_{z}
\end{array}\right]}
\end{aligned}
$$

we Observe that (4.8) is the transpose of (4.6), and (4.9) is the transpose of (4.7)

Where

Problem.1

$$
[0, \vec{u}]=\boldsymbol{Q}^{-1} \boldsymbol{p} \boldsymbol{Q}
$$

This publication is licensed under Creative Commons Attribution CC BY

http://dx.doi.org/10.29322/IJSRP.11.02.2021.p11040
Consider a rotation about an axis defined by $(1,1,1)$ through angle $2 \pi / 3$ about the axis .

We define a unit vector $\hat{u}=\frac{1}{\sqrt{3}}(1,1,1)$

Let rotation angle $\theta=2 \pi / 3$, then $\mathrm{Q}$ of rotation define as: $Q=$ $\cos 1 / 2 \theta+\sin 1 / 2 \theta \hat{v}$

$Q=\left[1 / 2, \frac{\sqrt{3}}{2} \times \frac{1}{\sqrt{3}}(1,1,1)\right]=[1 / 2,1 / 2(1,1,1)]$, then

$L_{Q}=\left[\begin{array}{lll}0 & 0 & 1 \\ 1 & 0 & 0 \\ 0 & 1 & 0\end{array}\right]$, let us compute the effect of rotation on the basic vector $i=(1,0,0)$, we obtain the resulting vector

$L_{Q} \overrightarrow{\mathrm{v}}=L_{Q} i=\left[\begin{array}{lll}0 & 0 & 1 \\ 1 & 0 & 0 \\ 0 & 1 & 0\end{array}\right]\left[\begin{array}{l}1 \\ 0 \\ 0\end{array}\right]=\left[\begin{array}{l}0 \\ 1 \\ 0\end{array}\right]=j$

Problem.2

To rotate point $\mathrm{P}(0,1,1)$ about $y$ - axis through angle $\theta=90^{\circ}$.

The $\mathrm{Q}$ of rotation define as:

$Q=\cos 1 / 2 \theta+\sin 1 / 2 \theta \hat{v}=\left[\frac{\sqrt{2}}{2}, \frac{\sqrt{2}}{2} j\right]$

Substituting $w=\frac{\sqrt{2}}{2}, x=0, y=\frac{\sqrt{2}}{2}, z=0$

There fore

$L_{Q} \overrightarrow{\mathrm{u}}=\left[\begin{array}{ccc}0 & 0 & 1 \\ 0 & 1 & 0 \\ -1 & 0 & 0\end{array}\right]\left[\begin{array}{l}0 \\ 1 \\ 1\end{array}\right]=\left[\begin{array}{l}1 \\ 1 \\ 0\end{array}\right]=i+j$

Matrix Method

It is obvious that $L_{Q^{*}}=L_{Q}^{T}$, the transpose of $L_{Q}$, and similarly, $R_{P^{*}}=R_{P}^{T}$.

Since $Q Q^{*}=|Q|$, the matrix $L_{Q} L_{Q}^{T}$ is the diagonal matrix $|Q| I$ (where $I$ is the identity $4 \times 4$ matrix), and similarly the matrix $R_{P} R_{P}^{T}$

is the diagonal matrix $|P| I$. Since $L_{Q}$ and $L_{Q}^{T}$ have the same determinant, we deduce that

$\operatorname{det}\left(L_{Q}\right)^{2}=|Q|^{4}$, and thus

$\operatorname{det}\left(L_{Q}\right)= \pm|Q|^{2}$. Then

$\operatorname{det}\left(L_{Q}\right)=w^{2}+x^{2}+y^{2}+z^{2}$

This shows that when $Q$ is a unit quaternion, $L_{Q}$ is a rotation matrix, and similarly when $P$ is a unit quaternion, $R_{P}$ is a rotation matrix

$\boldsymbol{u}$ Define the map $\varphi: \mathbb{H} \times \mathbb{H} \rightarrow \mathbb{R}$ as follows:

$\boldsymbol{u}_{\boldsymbol{y}} \boldsymbol{y}(Q, P)=1 / 2 \operatorname{Tr}(Q \bar{P})$

$\left.\boldsymbol{u}_{z}\right]_{\text {It }}=w w^{\prime}+x x^{\prime}+y y^{\prime}+z z^{\prime}$
It is easily verified that $\varphi$ is bilinear, symmetric, and definite positive. Thus, the quaternions form a Euclidean space under the inner product defined by $\varphi$ (see Berger [12], Dieudonn' e [46], Bertin [15]).

It is immediate that under this inner product, the norm of a quaternion $Q$ is just $\sqrt{|Q|}$. As a Euclidean space, His isomorphic to $E^{4}$. It is also immediate that the subspace $\mathbb{H}_{p}$ of pure quaternions is orthogonal to the space of "real quaternions" $\mathbb{R} 1$. The subspace $\mathbb{H}_{p}$ of pure quaternions inherits a Euclidean structure, and this subspace is isomorphic to the Euclidean space $E^{3}$. Since Hand $E^{4}$ are isomorphic Euclidean spaces, their groups of rotations $S O(\mathbb{H})$ and $S O(4)$ are isomorphic, and we will identify them.Similarly, we will identify $S O\left(\mathbb{H}_{p}\right)$ and $S O(3)$.

Quaternions and Rotations in $\mathrm{SO}(3)$

The group of $S O$ (3) is isomorphic to the group $\mathrm{U}(2)$ of quaternions $\exp (\hat{\mathrm{v}} \theta)=\cos \theta+\hat{\mathrm{v}} \sin \theta$ of unit length. 
Consider the sphere $S^{3}$ in $\mathbb{R}^{4}$, and special unitary $2 \times 2$ martix $S U(2)$

Recall that the 3 -sphere $S^{3}$ is the set of points $(x, y, z, t) \in \mathbb{R}^{4}$ such that

$$
x^{2}+y^{2}+z^{2}+t^{2}=1
$$

and that the real projective space $\mathbb{R} P^{3}$ is the quotient of $S^{3}$ modulo the equivalence relation that identifies antipodal points (where $(x, y, z, t)$ and $(x,-y,-z,-t))$ are antipodal points).

The group $S O(3)$ of rotations of $\mathbb{R}^{3}$ is intimately related to the 3 sphere $S^{3}$ and to the real projective space $\mathbb{R} P^{3}$. The key to this relationship is the fact that rotations can be represented by quaternions, discovered by Hamilton in 1843. Historically, the quaternions were the first instance of a skew field. As we shall see, quaternions represent rotations in $\mathbb{R}^{3}$. Then every plane rotation $\rho \theta$ by an angle $\theta$ is represented by multiplication by the quaternion $\exp (\hat{\mathrm{v}} \theta) \in S U(2)$, in the sense that for all $Q, Q^{\prime} \in \mathbb{H}: Q^{\prime}=$ $\rho \theta(Q)$ iff $Q^{\prime}=\exp (\hat{\mathrm{v}} \theta) Q$

If $Q=x i+y j+z k$ and $P=x^{\prime} i+y^{\prime} j+z^{\prime} k$ are pure quaternions, identifying $Q$ and $P$ with the corresponding vectors in $\mathbb{R}^{3}$,the inner product $Q \cdot P$ and the cross product $Q \times P$ make sense, and letting $[0, Q \times P]$ denote the quaternion whose first component is 0 and whose last three components are those of $Q \times$ $P$, we have the remarkable identity

$$
\mathrm{QP}=-(Q \cdot P) 1+[[0, Q \times P](4.13)
$$

More generally, given quaternion

$Q=t 1+x i+y j+z k$, we can write it

as $=[w,(x, y, z)]$, where $w$ is called the scalar part of $Q$ and $(x, y, z)$ thepure part of $Q$. Then if $Q=[w, \overrightarrow{\mathrm{v}}]$ and $P=\left[w^{\prime}, \overrightarrow{\mathrm{v}}^{\prime}\right]$ it is easily seen that the quaternion product $Q P$ can be expressed as

$$
Q P=\left[w w^{\prime}-\overrightarrow{\mathrm{v}} \cdot \overrightarrow{\mathrm{v}}^{\prime}, w \overrightarrow{\mathrm{v}}^{\prime \prime}+w^{\prime} \overrightarrow{\mathrm{v}}+\overrightarrow{\mathrm{v}} \times \overrightarrow{\mathrm{v}}^{\prime}\right](4.14)
$$

The above formula for quaternion multiplication allows us to show the

following fact. Let $Z \in \mathbb{H}$, and assume that $Z Q=Q Z$ for all $Q \in$ $\mathbb{H}$.We claim that the pure part of $Z$ is null, i.e., $Z=w 1$ for some $w \in \mathbb{R}$.

Indeed, writing $Z=[w, \overrightarrow{\mathrm{u}}]$, if $\overrightarrow{\mathrm{u}} \neq 0$

there is at least one non null pure quaternion $Q=[0, \overrightarrow{\mathrm{v}}]$ such that $\overrightarrow{\mathrm{u}} \times \overrightarrow{\mathrm{v}} \neq 0$.

For arbitrary quaternions $Q=[w, \overrightarrow{\mathrm{v}}]$ and

$P=\left[w, \overrightarrow{\mathrm{v}}^{\prime}\right]$, satisfies Lie algebra

$$
[Q, P]=Q P-P Q=\left[0,2\left(\overrightarrow{\mathrm{v}} \times \overrightarrow{\mathrm{v}}^{\prime}\right)\right]
$$

and that for pure quaternions $Q, P \in \mathbb{H}_{p}$

$$
2(Q \cdot P)) 1=-(Q P+P Q) \text {. }
$$

Since quaternion multiplication for a given $Q$ is bilinear, the map $P \mapsto Q P$ is linear, and similarly for a given $P$, the map $Q \mapsto Q P$ is linear. It is immediate that if the matrix of the first map is $L_{Q}$ and the matrix of the second map is $R_{P}$, then

$$
Q P=L_{q} P=\left[\begin{array}{cccc}
w & -x & -y & -z \\
x & w & z & -y \\
y & -z & w & x \\
z & y & -x & w
\end{array}\right]\left[\begin{array}{c}
w^{\prime} \\
x^{\prime} \\
y^{\prime} \\
z^{\prime}
\end{array}\right]
$$

and $Q P=$

$$
R_{p} Q=\left[\begin{array}{cccc}
w^{\prime} & -x^{\prime} & -y^{\prime} & -z^{\prime} \\
x^{\prime} & w^{\prime} & z^{\prime} & -y^{\prime} \\
y^{\prime} & -z^{\prime} & w^{\prime} & x^{\prime} \\
z^{\prime} & y^{\prime} & -x^{\prime} & w^{\prime}
\end{array}\right]\left[\begin{array}{l}
w \\
x \\
y \\
z
\end{array}\right]
$$

We observe that the columns (and the rows) of the above matrices are orthogonal. Thus, when $\mathrm{Q}$ and $\mathrm{P}$ are unit quaternions, both $\mathrm{L}_{\mathrm{Q}}$ and $\mathrm{R}_{\mathrm{P}}$ are orthogonal matrices .

Multiple Rotations

Say a vector or frame of reference is subjected to two rotations specified by $Q_{1}$

followed by $Q_{2}$. There is a temptation to convert both quaternions to their respective

matrix and multiply the matrices together. However, this not the most efficient way

of combining the rotations. It is best to accumulate the rotations as quaternions and

then convert to matrix notation, if required.

To illustrate this, consider the pure quaternion $P$ subjected to the first quaternion $Q_{1}$

$$
Q_{1} P Q_{1}^{*}
$$

followed by a second quaternion $Q_{2}$

which can be expressed as

$$
Q_{2}\left(Q_{1} P Q_{1}^{*}\right) Q_{2}^{*}
$$

$$
Q_{2}\left(Q_{1} P Q_{1}^{*}\right) Q_{2}^{*}=\left(Q_{2} Q_{1}\right) P\left(Q_{2} Q_{1}\right)^{*}
$$

Extra quaternions can be added accordingly.

$$
\begin{gathered}
Q_{3}\left[Q_{2}\left(Q_{1} P Q_{1}^{*}\right) Q_{2}^{*}\right] Q_{3}^{*}=Q_{3}\left[\left(Q_{2} Q_{1}\right) P\left(Q_{2} Q_{1}\right)^{*}\right] Q_{3}^{*} \\
Q_{3}\left(Q_{2} Q_{1}\right) P\left(Q_{2} Q_{1}\right)^{*} Q_{3}^{*}=\left(Q_{3} Q_{2} Q_{1}\right) P\left(Q_{3} Q_{2} Q_{1}\right)^{*} \\
Q_{3}\left[Q_{2}\left(Q_{1} P Q_{1}^{*}\right) Q_{2}^{*}\right] Q_{3}^{*}=\left(Q_{3} Q_{2} Q_{1}\right) P\left(Q_{3} Q_{2} Q_{1}\right)^{*}
\end{gathered}
$$

\section{Eigenvalue and Eigenvector}

Although there is no doubt that (4.6) is a rotation matrix, we can secure further

evidence by calculating its eigenvalue and eigenvector. The eigenvalue should be $\theta$

where

$$
\begin{aligned}
& \operatorname{Tr}\left(Q \overrightarrow{\mathrm{v}} Q^{-1}\right) \\
& =\mathbf{1}-\mathbf{2}\left(y^{2}+z^{2}\right)+\mathbf{1}-\mathbf{2}\left(x^{2}+z^{2}\right)+\mathbf{1}-\mathbf{2}\left(x^{2}+y^{2}\right) \\
& =4 w^{2}+2\left(w^{2}+x^{2}+y^{2}+z^{2}\right)-3=4 w^{2}-1 \\
& =4 \cos ^{2} 1 / 2 \theta-1=4 \cos \theta+4 \sin ^{2} 1 / 2 \theta-1 \\
& =4 \cos \theta+2-2 \cos \theta-1=1+2 \cos \theta
\end{aligned}
$$

and

$\cos \theta=1 / 2\left(\operatorname{Tr}\left(Q \overrightarrow{\mathrm{v}} Q^{-1}\right)-1\right)$

To compute the eigenvector of (4.6) we use the three equations

$$
\begin{aligned}
& x_{v}=\left(a_{22}-1\right)\left(a_{33}-1\right)-a_{23} a_{32} \\
& y_{v}=\left(a_{11}-1\right)\left(a_{33}-1\right)-a_{13} a_{31} \\
& z_{v}=\left(a_{11}-1\right)\left(a_{22}-1\right)-a_{12} a_{21}
\end{aligned}
$$

Therefore,

$x_{v}=4 x^{2}, y_{v}=4 y^{2}$ and $z_{v}=4 z^{2}$, which confirm that the eigenvector has components associated with the quaternion's vector. The square terms should be no surprise, as the triple $Q P Q^{-1}$

includes the product of three quaternions.

\section{Frames of Reference}

The product $Q P Q^{-1}$ is used for rotating points about the vector associated with the

Quaternion $\mathrm{Q}$, whereas the triple $Q^{-1} P Q$ can be used for rotating points about the

same vector in the opposite direction.

But this reverse rotation is also equivalent

to a change of frame of reference. 


\section{Interpolating Quaternions}

Like vectors, quaternions can be interpolated to compute an inbetween quaternion.

However, whereas two interpolated vectors results in a third vector that is readily

visualised, two interpolated quaternions results in a third quaternion that acts as a

rotor, and is not immediately visualised.

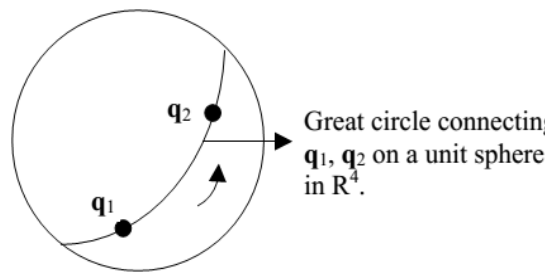

Fig. 4 Spherical linear

\section{interpolation between two unit}

The spherical interpolant for vectors is

$$
\overrightarrow{\mathrm{v}}=\frac{\sin (1-t) \theta}{\sin \theta} \mathrm{v}_{1}+\frac{\sin s \theta}{\sin \theta} \mathrm{v}_{2}
$$

where $\theta$ is the angle between the vectors, The spherical linear interpolation between $Q_{1}$ and $Q_{2}$ is given by: $\operatorname{Slerp}\left(Q_{1}, Q_{2} ; t\right)$

So, given

$$
Q=\frac{\sin (1-t) \theta}{\sin \theta} \mathrm{Q}_{1}+\frac{\sin s \theta}{\sin \theta} \mathrm{Q}_{2}, 0 \leq t<1
$$

$$
\begin{aligned}
& \mathrm{Q}_{1}=\left[w_{1}, x_{1} i+y_{1} j+z_{1} k\right] \\
& \mathrm{Q}_{2}=\left[w_{2}, x_{2} i+y_{2} j+z_{2} k\right]
\end{aligned}
$$

$\theta$ is obtained by taking the $4 \mathrm{D}$ dot product of $\mathrm{Q}_{1}$ and $\mathrm{Q}_{2}$ :

$$
\begin{aligned}
& \cos \theta=\frac{\mathrm{Q}_{1} \mathrm{Q}_{2}}{\left|\mathrm{Q}_{1}\right|\left|\mathrm{Q}_{2}\right|}(4.21) \\
& \cos \theta=\frac{w_{1} w_{2}+x_{1} x_{2}+y_{1} y_{2}+z_{1} z_{2}}{\left|\mathrm{Q}_{1}\right|\left|\mathrm{Q}_{2}\right|}
\end{aligned}
$$

and if we are working with unit-norm quaternions, then

$$
\cos \theta=w_{1} w_{2}+x_{1} x_{2}+y_{1} y_{2}+z_{1} z_{2}
$$

\section{Converting a Rotation Matrix to a Quaternion}

The matrix transform equivalent to $q p q^{-1}$

is:

$$
\begin{aligned}
& Q P Q^{-1} \\
& =\left[\begin{array}{ccc}
\mathbf{1}-\mathbf{2}\left(y^{2}+z^{2}\right) & \mathbf{2}(x y-\boldsymbol{w} z) & \mathbf{2}(x z+\boldsymbol{w} y) \\
\mathbf{2}(x y+\boldsymbol{w} z) & \mathbf{1}-\mathbf{2}\left(x^{2}+z^{2}\right) & \mathbf{2}(y z-\boldsymbol{w} x) \\
\mathbf{2}(x z-\boldsymbol{w} y) & \mathbf{2}(y z+\boldsymbol{w} x) & \mathbf{1}-\mathbf{2}\left(x^{2}+y^{2}\right)
\end{array}\right] \\
& =\left[\begin{array}{lll}
a_{11} & a_{12} & a_{13} \\
a_{21} & a_{22} & a_{23} \\
a_{31} & a_{32} & a_{33}
\end{array}\right] \quad(4.22 .1)
\end{aligned}
$$

Inspection of the matrix shows that by combining various elements we can isolate

the terms of a quaternion $t, x, y, z$. For example, by adding the terms

$a_{11}+a_{22}+a_{33}$ we obtain

therefore,

$$
a_{11}+a_{22}+a_{33}=4 t^{2}-1
$$

$t= \pm 1 / 2 \sqrt{1+a_{11}+a_{22}+a_{33}}$

To find $x, y$ and $z$ we use

$$
\begin{aligned}
& x=\frac{1}{4 t}\left(a_{32}-a_{23}\right) \\
& y=\frac{1}{4 t}\left(a_{13}-a_{31}\right) \\
& z=\frac{1}{4 t}\left(a_{21}-a_{12}\right)
\end{aligned}
$$

\section{Euler Angles to Quaternion}

Perhaps the most important property of quaternions isthat they can characterize rotations in a three dimensional space. The conventional way of representing three-dimensional rotations is by using a set of Euler angles $\{\psi, \varphi, \theta\}$ which denote rotations about independent coordinate axes. The function that maps this transformation to its corresponding rotation matrix $\boldsymbol{R}_{z y x}: \mathbb{R}^{3} \rightarrow$ $S O(3)$ is:[8]

$\vec{u}=Q_{z} Q_{y} Q_{x} \vec{v}=R_{\psi, z} R_{\varphi, y} R_{\theta, x} \vec{v}$

$\left[\begin{array}{l}x^{\prime} \\ y^{\prime} \\ z^{\prime}\end{array}\right]$

$=\left[\begin{array}{ccc}\cos \psi & -\sin \psi & 0 \\ \sin \psi & \cos \psi & 0 \\ 0 & 0 & 1\end{array}\right]\left[\begin{array}{ccc}\cos \varphi & 0 & \sin \varphi \\ 0 & 1 & 0 \\ -\sin \varphi & 0 & \cos \varphi\end{array}\right]\left[\begin{array}{ccc}1 & 0 & 0 \\ 0 & \cos \theta & -\sin \theta \\ 0 & \sin \theta & \cos \theta\end{array}\right]\left[\begin{array}{l}x \\ y \\ z\end{array}\right]$

(4.23)[3]

Expanding, we find $w, x^{\prime}, y^{\prime}$ and $z^{\prime}$, we get

$w=\cos 1 / 2 \psi \cos 1 / 2 \varphi \cos 1 / 2 \theta+\sin 1 / 2 \psi \sin 1 / 2 \varphi \sin 1 / 2 \theta$

$x^{\prime}=\cos 1 / 2 \psi \cos 1 / 2 \varphi \sin 1 / 2 \theta-\sin 1 / 2 \psi \sin 1 / 2 \varphi \cos 1 / 2 \theta$

$y^{\prime}=\cos 1 / 2 \psi \sin 1 / 2 \varphi \cos 1 / 2 \theta-\sin 1 / 2 \psi \cos 1 / 2 \varphi \sin 1 / 2 \theta$

$z^{\prime}=\sin 1 / 2 \psi \cos 1 / 2 \varphi \cos 1 / 2 \theta-\cos 1 / 2 \psi \sin 1 / 2 \varphi \sin 1 / 2 \theta$

where

$q=\left[w, x^{\prime} i+y^{\prime} j+y^{\prime} k\right]$

And the three rotation transforms

$\begin{aligned} R_{\theta, x} & =\left[\begin{array}{ccc}1 & 0 & 0 \\ 0 & \cos \theta & -\sin \theta \\ 0 & \sin \theta & \cos \theta\end{array}\right] \\ R_{\Phi, y} & =\left[\begin{array}{ccc}\cos \varphi & 0 & \sin \varphi \\ 0 & 1 & 0 \\ -\sin \varphi & 0 & \cos \varphi\end{array}\right] \\ R_{\psi, z} & =\left[\begin{array}{ccc}\cos \psi & -\sin \psi & 0 \\ \sin \psi & \cos \psi & 0 \\ 0 & 0 & 1\end{array}\right]\end{aligned}$

Then

$R_{\psi, z}, R_{\varphi, y}, R_{\theta, x}$

$=\left[\begin{array}{ccc}\mathrm{C} \psi \mathrm{C} \varphi & -\mathrm{S} \psi \mathrm{C \theta}+\mathrm{C} \psi \mathrm{S} \varphi \mathrm{S} \theta & \mathrm{S} \psi \sin \theta+\mathrm{C} \psi \mathrm{S} \varphi \mathrm{C} \theta \\ \mathrm{S} \psi \cos \varphi & \mathrm{C} \psi \mathrm{C \theta}+\mathrm{S} \psi \mathrm{S} \varphi \mathrm{S} \theta & -\mathrm{C} \psi \mathrm{S} \theta+\mathrm{S} \psi \mathrm{S} \varphi \mathrm{C \theta} \\ -\mathrm{S} \varphi & \mathrm{C} \varphi \mathrm{S} \theta & \mathrm{C} \varphi \mathrm{C} \theta\end{array}\right]$ (4.27)

Then the most general rotation of a rigid body with a fixed point can be achieved by a single rotation about an axis through the fixed point. If $v=(l, m, n)$ denote a vector in 3-D space, then a rotational transformation by an angle $\theta$ about this vector is given by [3]

$\left[\begin{array}{l}x^{\prime} \\ y^{\prime} \\ z^{\prime}\end{array}\right]$

$=\left[\begin{array}{ccc}l^{2}(1-C \theta)+C \theta & \operatorname{lm}(1-C \theta)-n S \theta & n l(1-C \theta)+m S \theta \\ l m(1-C \theta)+n S \theta & m^{2}(1-C \theta)+C \theta & m n(1-C \theta)-l S \theta \\ n l(1-C \theta)-m S \theta & m n(1-C \theta)+l S \theta & n^{2}(1-C \theta)+C \theta\end{array}\right]$ (5.28)

Where, $\vec{u}=\left(x^{\prime}, y^{\prime}, z^{\prime}\right), \overrightarrow{\mathrm{v}}=(x, y, z)$ and

$Q=(\cos 1 / 2 \theta+l \sin 1 / 2 \theta++m \sin 1 / 2 \theta+n \sin 1 / 2 \theta)$ 


\section{CONCLUSION}

In this paper, compact overview of mathematical constructs used most often quaternion rotation operator acting on any vector $\vec{v}$ in the three-dimensional space. Thisoperator plays an important role in classical mechanics as well as in computer graphics.

Vector transformations using unit quaternions are presented. These transformations are used as foundation for new, simple and compact direct kinematic algorithm in unit quaternion space.

Developed algorithm is demonstrated on human centrifuge which is modelled as 3 dimentional of manipulator with rotational joints.

\section{REFERENCES}

[1] [1]William Rowan Hamilton, ON QUATERNIONS, OR ON A NEW SYSTEM OF IMAGINARIES IN ALGEBRA, (Philosophical Magazine, (1844-1850))

[2] [2] Ryan T.Flynn, QUATERNION ALGEBRAS AND ELLIPTIC CURVES OVER FUNCTION FIELDS OF FINITE CHARACTERISTIC, The Pennsylvania State University, August 2013.

[3] [3] R.Mukunda, Quaternions: From Classical Mechanics to Computer Graphics, and Beyond, University of Canterbury Christchurch, New Zealand, Conference in Mathematics 2002. Invited Paper.

[4] [4] Mehdi Jafari ,ON THE MATRIX ALGEBRA OF COMPLEX QUATERNIONS-16-09-2013, Urmia University 64 PUBLICATIONS 204 CITATIONS Urmia University August 2015.
[5] [5] John Vince, Quaternions for Coumputer Graphics, Springer

[6] [6] Johannes C. Familton, QUATERNIONS A HISTORY OF COMPLEX NONCOMMUTATIVE ROTATION GROUPS IN THEORETICAL PHYSICS, Columbia University,2015.

[7] [7] Jernej T. Barbic, Quaternions and Rotations, University of Southern California, CSCI 520 Computer Animation and Simulation.

[8] [8] Jelena-Z.Vidakovic and others, Advanced Quaternion Forward Kinematics Algorithm Including Overview of Different Methods for Robot Kinematics, University of Belgrade Loia institute 198.Vol.42 No3 2014

[9] [9] Jean Gallier, Clifford Algebras-Clifford Groups and a Generalization of the Quaternions ,University of Pennsylvania Philadelphia, PA 1104, USA, January 31,2012

[10] [10] Dominic Widdows, Quaternion Algebraic Geometry, June 5, 2006.

[11] [11] Basile Graf, Quaternions And Dynamics , February, 2007.

[12] [12] Arthur S.Hthaway, QUATERNION SPAC, Rose Polytechnic Institute,Terre Haute, Ind. paper published in( vol. 2, p. 183)

[13] 13] Ismail Mustafa Mohammed, QURTERNIONS ,Journal of Economic \&Political and statistics Sciences, Omdurman Islamic University, No 15 ISSN:1858-5876 2013

[14] [14] Erik B and others, Quaternions, Interpolation and Animation, Technical Report DIKU-TR98/5,Department of Computer Sciences -University of Copenhagen ,July 171998

\section{AUTHORS}

First Author - Emran Sasi Althabit*, PH.D of mathematics, SUDAN UNIVERISITY SEEINCE AND TECHNOLOGY

(Khartoum), omransasi045@gmail.com +218926083695

Second Author - Ismail Mustafa Mohamed**, PH.D in mathematics, AL NEELAN UNIVERISITY (Khartoum), abuahd91@gmail.com ,+249124699207 